\title{
Timing the release in sequential double ionization
}

\author{
Adrian N. Pfeiffer ${ }^{1 \star}$, Claudio Cirelli ${ }^{1}$, Mathias Smolarski ${ }^{1}$, Reinhard Dörner ${ }^{2}$ and Ursula Keller ${ }^{1}$
}

\begin{abstract}
The timing of electron release in strong-field double ionization poses great challenges both for conceptual definition and for conducting experimental measurements. Here we present coincidence momentum measurements of the doubly charged ion and of the two electrons arising from double ionization of argon using elliptically polarized laser pulses. Based on a semi-classical model, the ionization times are calculated from the measured electron momenta across a large intensity range. This paper discusses how this method provides timings on a coarse and on a fine scale, similar to the hour and the minute hand of a clock. We found that the ionization time of the first electron is in good agreement with the simulation, whereas the ionization of the second electron occurs significantly earlier than predicted.
\end{abstract}

A mong all the methods used to measure time, one of the most fundamental is to measure the angle of a rotating hand, such as is done on an analogue watch face. This principle can be employed in strong-field ionization using laser pulses with close-to-circular polarization. In the attoclock the rotating electric field vector is used to deflect photo-ionized electrons, such that the instant of ionization is mapped to the final angle of the momentum, similar to the minute hand of a clock. The attoclock technique is based on the definition of 'time' by 'counting cycles' ${ }^{1,2}$. During one period the watch hand completes one cycle, such that measuring the emission angle of the electron enables us to measure time at a precision well below one optical period ${ }^{2}$. Thus the measurement provides attosecond timing without using an attosecond pulse.

Here we use the attoclock to measure the ionization times of the two electrons in the double ionization of argon. As a result of depletion the averaged ionization time of the electrons is shifted towards the beginning of the pulse, thus requiring a multi-cycle measurement. The magnitude of the electron momenta follows the envelope of the laser pulse and gives a coarse timing for the electron release (that is 'the hour hand of the clock'). The emission angle of the electrons subsequently gives the fine timing (that is 'the minute hand of the clock').

The result of the attoclock measurements addresses a fundamental question in double ionization: are there electron correlation mechanisms that are not induced by recollision? With linearly polarized fields in strong-field double ionization the dominating ionization mechanism is induced by recollision of the first emitted electron $^{3,4}$. With close-to-circular polarization, however, we can avoid this recollision and therefore investigate a conceptually even simpler process of few-body quantum mechanics.

It is impossible, at present, to simulate this process based on the time dependent Schrödinger equation (TDSE) because of exceedingly large computing time requirements ${ }^{5}$. Instead one describes the process usually in terms of simplifying mechanisms, which can be classified as sequential double ionization (SDI) or non-sequential double ionization (NSDI; ref. 6). For circular polarization, only SDI is usually believed to be relevant.

We use the term SDI to include two approximations. First, the assumption of two independent and successive electron release steps, and second, tunnel ionization for the individual steps. The validity of these models has to be probed making a comparison of adequate observables in simulation and experiment. In this work we find that the ionization time both for the first and the second ionization step is shifted towards the beginning of the pulse with increasing intensity, in accordance with the SDI model that includes depletion. The absolute ionization time is predicted well by this SDI model for the first ionization step, but the second ionization occurs earlier than predicted by tunnelling theory.

The paper is organized as follows. First we present the structure of the ion momentum distribution and show a characteristic bifurcation from a 3-peak structure into a 4-peak structure. This can be explained by depletion of the neutral argon atoms. Thereafter, we discuss how the ionization times can be extracted from the electron momenta on the basis of a simple semi-classical model.

\section{Bifurcation of the ion momentum distribution}

We use a Ti:sapphire-based laser system to double ionize argon atoms in a cold gas jet and measure the momenta of the ions and electrons by Cold Target Recoil Ion Momentum Spectroscopy (COLTRIMS) (see Methods section). Figure 1 depicts the ion momentum distribution in the polarization plane, which is perpendicular to the laser propagation direction ( $z$ axis). Along the major axis of the polarization ellipse ( $y$ axis), the momentum distribution is close to Gaussian, whereas along the minor axis ( $x$ axis) a peak structure shows up. We could rule out that this is an artefact of anisotropic momentum resolution (about 0.3 a.u. for $p_{x}$ and 3 a.u. for $p_{y}$ ) by rotating the polarization ellipse.

The origin of the peak structure was explained by Maharjan et al. ${ }^{7}$ within a semi-classical model: ionization occurs preferentially when the electric-field vector points along the major polarization axis ( $y$ axis), producing electrons with a momentum pointing in the $x$ direction after acceleration by the electric field of the laser pulse ${ }^{8}$. The situation where the electrons are ejected into anti-parallel directions causes the ion to stay at close to zero momentum (centre peak), whereas parallel electron ejection results in the side peaks of the momentum distribution. Interestingly, the peak structure can also be explained with a fully-classical model ${ }^{9}$.

The projection of the momentum distribution onto the $x$ axis exhibits a characteristic dependence on intensity: the peak structure undergoes a bifurcation from a 3-peak structure at low intensities to a 4 -peak structure at high intensities. To understand this bifurcation we model the process semi-classically following

${ }^{1}$ Physics Departement, ETH Zurich, CH-8093 Zürich, Switzerland, ${ }^{2}$ Institut für Kernphysik, Johann Wolfgang Goethe Universität, Max-von-Laue-Str. 1 , 60438 Frankfurt am Main, Germany. ${ }^{\star}$ e-mail: apfeiff@phys.ethz.ch. 


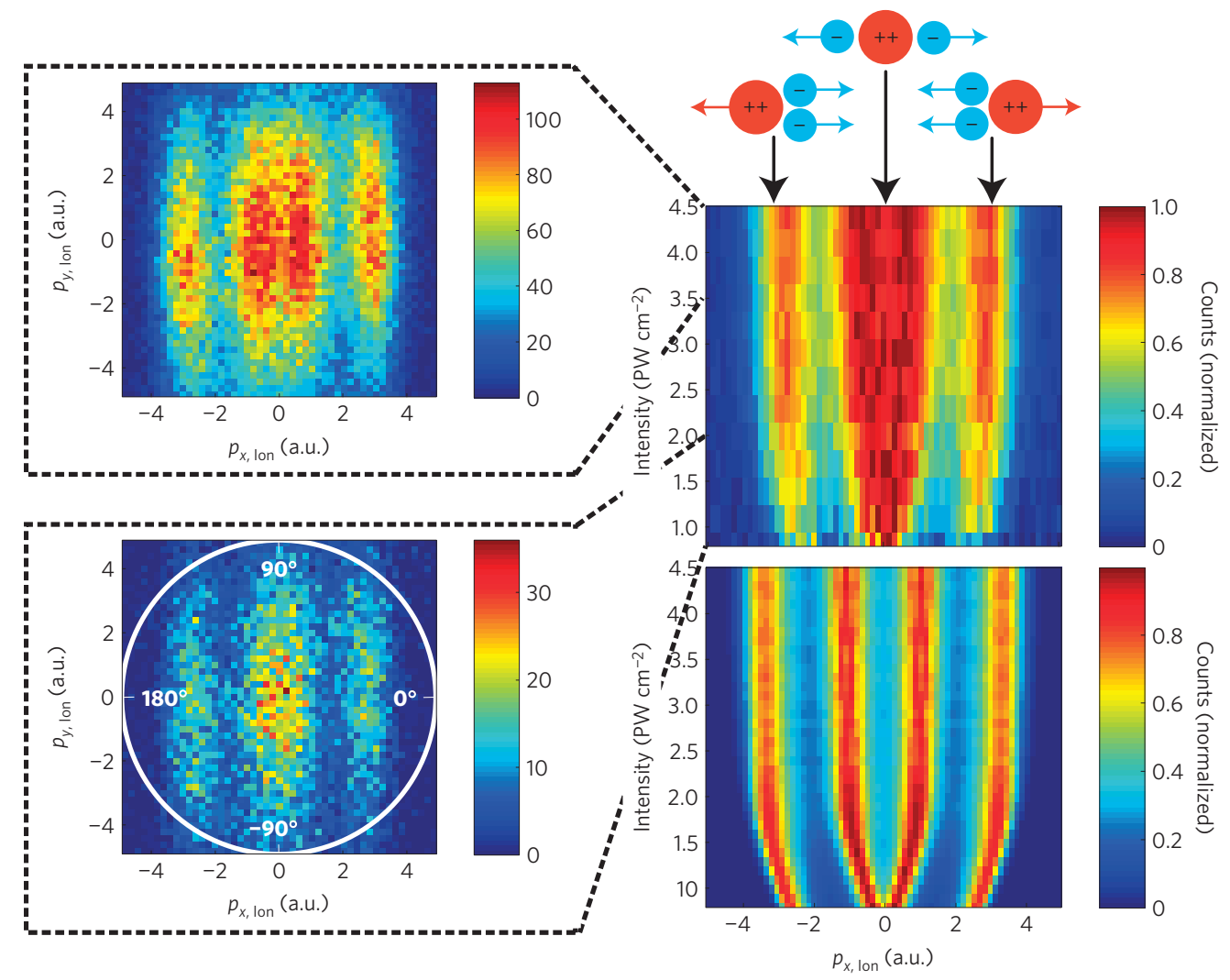

Figure 1 | Bifurcation of the ion momentum distribution. Momentum distribution of the $\mathrm{Ar}^{++}$ions for the 33-fs laser pulse as measured with a COLTRIMS set-up for low intensities (bottom left) and high intensities (top left). The dashed lines indicate the range of intensity over which the data are integrated. The projection onto the $x$ axis as a function of intensity is depicted on the right (top: data, bottom: SDI simulation with option (1), see Simulation details). To enhance visibility over a large intensity range, each line of the image is normalized.

the 3-step model of high-harmonic generation ${ }^{10}$ (see Simulation details). In the model it is assumed that the two electrons interact independently with the laser field. In a first step the electrons are released into the continuum according to a static field ionization rate. In the second step the electrons are accelerated in the electric field of the laser pulse starting with zero kinetic energy. The final electron momentum amplitude after the laser pulse follows the envelope of the electric field (Fig. 2a). At low intensities, where the maximum ionization rate is close to the pulse centre for both electrons, anti-parallel electron emission causes the ion to end up with zero momentum after the laser pulse. For higher intensities it is important to take depletion into account, which means that the ionization rate needs to be weighted with the survival probability of the neutral atoms ${ }^{11}$. Therefore the maximum ionization rate is reached before the pulse peak, because almost all atoms are already ionized before the peak. The averaged ionization time of the first electron occurs significantly before the second electron, which causes the momenta for anti-parallel electron emission not to cancel out completely. The simulation predicts a minimum at the centre of the momentum distribution once the intensity reaches a threshold value, qualitatively in agreement with the data (Fig. 1).

\section{Attoclock measurements of ionization times}

The semi-classical model described above opens up the possibility to use the measured momenta of the electrons to calculate the timing of the ionization process. If one knows the electric field of the pulse, one can trace back the classical electron trajectory in the electric field and determine the time when the trajectory was started ${ }^{1,2,8}$. Here we define the term ionization time for this instant of time. It is the time when the electron is released into the continuum with zero kinetic energy according to the semi-classical model.
By giving this definition we intend to avoid confusion with other times describing the ionization process, such as the tunnelling delay time $^{2}$, the electron release time $e^{12}$ or the delay in photoemission ${ }^{13}$, and stress the fact that the semi-classical ionization time is only meaningful within the framework of the semi-classical model.

Figure 2 describes the semi-classical ionization time measurement using the attoclock ${ }^{1,2}$. An electron that is released at a time $t$ is driven by the electric field of the laser pulse and obtains a momentum $\mathbf{p}(t)=-\mathbf{A}(t)$, where $\mathbf{A}$ is the vector potential (see Simulation details). For perfectly circular polarization the polar coordinates of the momenta (denoted by $p_{\mathrm{r}}$ and $p_{\varphi}$ ) can be divided into the hour hand and the minute hand. Both $p_{\mathrm{r}}$ and $p_{\varphi}$ carry information about the ionization time. The timing provided by $p_{\mathrm{r}}$ is rather coarse, but has the advantage that it is unambiguous if restricted to the time interval $[-\infty, 0]$, which can be assumed for high intensities because of depletion (time zero is defined by the peak of the pulse envelope, Fig. 2a). A fine timing with sub-cycle precision can be extracted from $p_{\varphi}$, with the disadvantage that the timing is unambiguous only if restricted to one laser period.

Assuming a Gaussian field envelope

$$
f(t)=E_{0} \exp \left(-\frac{1}{2}\left(\frac{t}{\sigma}\right)^{2}\right)
$$

the coarse estimate for the ionization time is approximated by

$$
t_{\text {coarse }}=-\sigma \sqrt{2 \ln \left(\frac{E_{0}}{p_{\mathrm{r}} \omega}\right)}
$$

Calculating the absolute ionization time from $p_{\varphi}$ requires knowledge of the absolute carrier-envelope-offset (CEO) phase ${ }^{14}$. 
a Coarse timing ('hour hand')
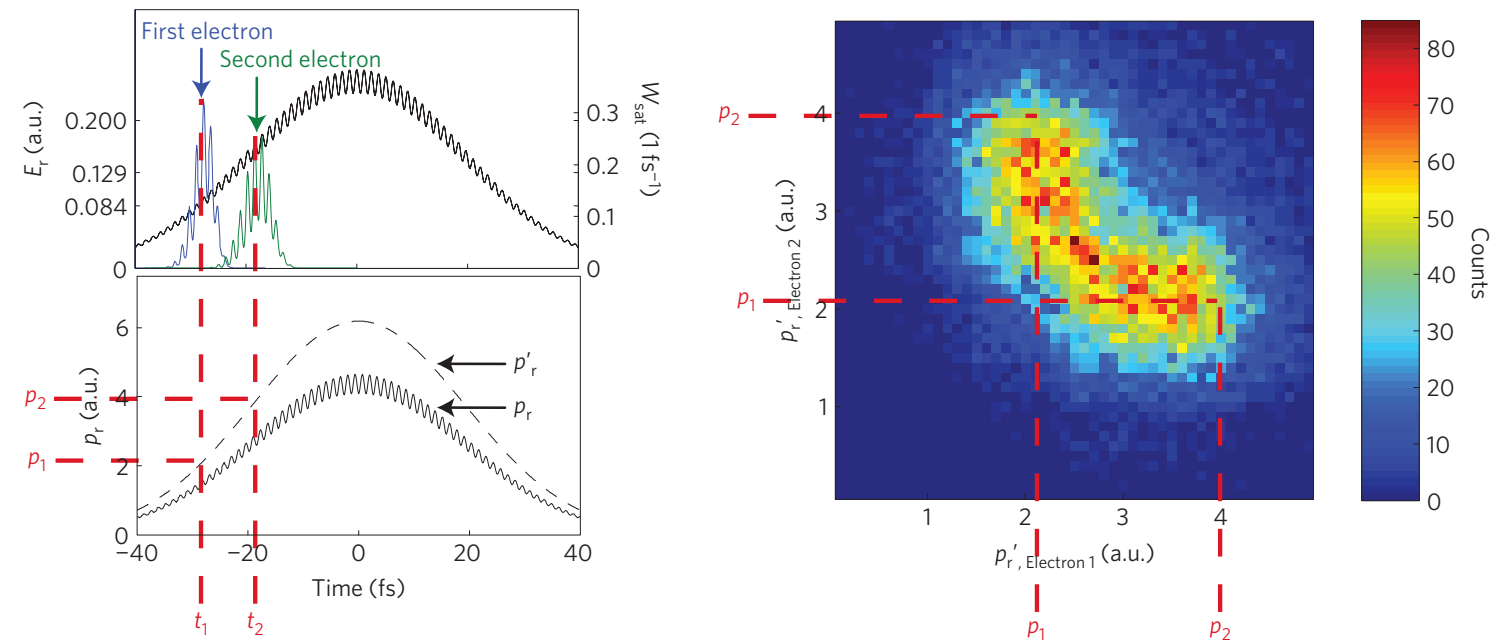

b Fine timing ('minute hand')
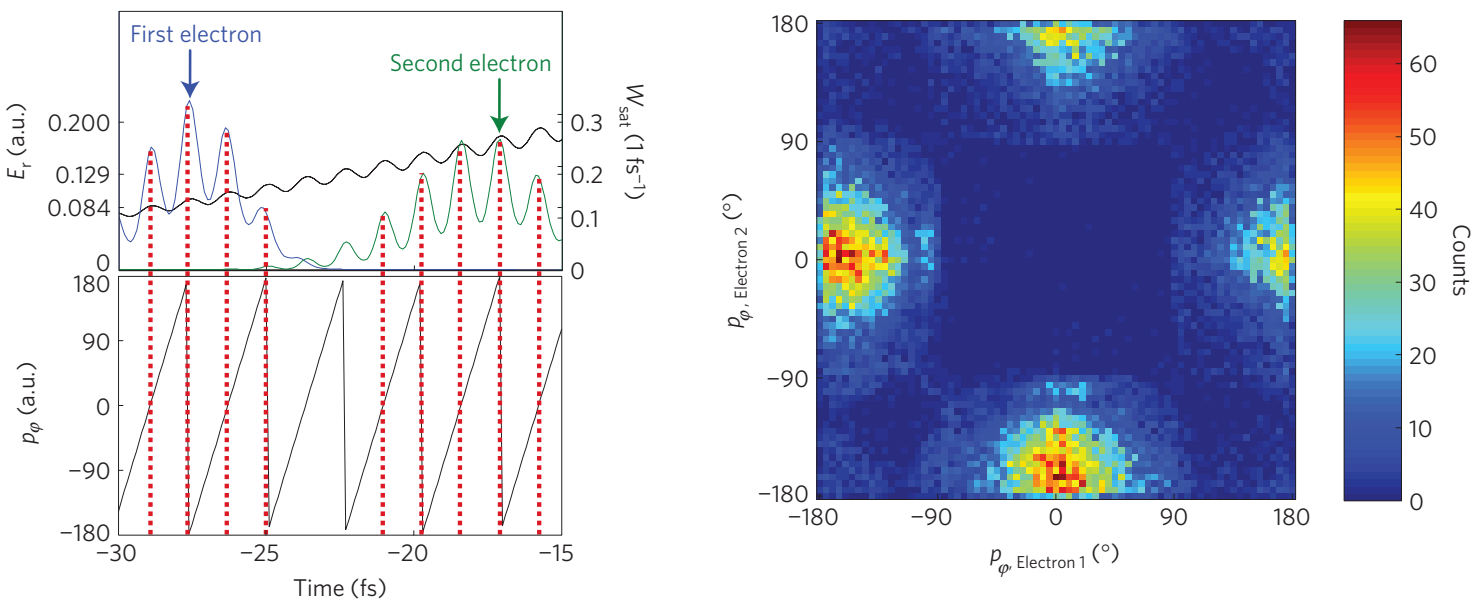

Figure 2 | The hands of the attoclock. a, 'Hour hand'. The ionization rate $W_{\text {sat }}$ of the first (around $t_{1}$ ) and the second (around $t_{2}$ ) ionization step is confined to a time interval at the beginning of the laser pulse. The radial electron momentum $p_{r}$ of an elliptically polarized Gaussian laser pulse is in general not an injective function of time, but the measurement of the ellipticity and the alignment of the polarization axis allows the calculation of $p_{r}^{\prime}=\left(\left(\left(\varepsilon^{2}+1\right) / \varepsilon^{2}\right) p_{x}^{2}+\left(\varepsilon^{2}+1\right) p_{y}^{2}\right)^{1 / 2}$, which is injective under the assumption that the electron is released before the peak of the pulse. The averaged release times $t_{1}$ and $t_{2}$ for the two consecutive ionization steps can be associated with momenta $p_{1}$ and $p_{2}$, which can be identified in the electron-electron correlation plot of $p_{r}$ '. $\mathbf{b}$, 'Minute hand'. Owing to the ellipticity, the ionization rate exhibits one peak per optical half cycle. The angle of the electron momentum $p_{\varphi}$ at these peaks is either $0^{\circ}$ or $\pm 180^{\circ}$, as indicated by the red dotted lines. The influence of the ellipticity on $p_{\varphi}$ is very small and cannot be seen in this plot. In the electron-electron correlation plot of $p_{\varphi}$ the peaks show up for anti-parallel electron emission at $\left( \pm 180^{\circ}, 0^{\circ}\right)$ and $\left(0^{\circ}, \pm 180^{\circ}\right)$. The peaks for parallel electron emission expected at $\left(0^{\circ}, 0^{\circ}\right)$ and $\left( \pm 180^{\circ}, \pm 180^{\circ}\right)$ are not detected. See Fig. 1 for the definition of angle. Laser parameters: intensity $=4.5 \mathrm{PW} \mathrm{cm}^{-2}$, ellipticity $=0.77, \mathrm{FWHM}=33 \mathrm{fs}$.

Nevertheless, also for experiments without CEO phase stabilization one can use the high temporal precision encoded in $p_{\varphi}$ for an estimate of the difference of the ionization times:

$$
t_{\text {fine }, 2}-t_{\text {fine }, 1}=\frac{p_{\varphi, 2}-p_{\varphi, 1}}{\omega}+n \frac{2 \pi}{\omega}
$$

where $n$ is an integer indicating the number of optical cycles between the two events. For the data presented in Fig. $3 \mathrm{c}$ and $\mathrm{d}, n$ is chosen to minimize the expression

$$
|| t_{\text {fine }, 2}-t_{\text {fine }, 1}|-| t_{\text {coarse }, 2}-t_{\text {coarse }, 1}||
$$

This combines the unambiguity of the coarse time measurement with the precision of the fine time measurement, and avoids at the same time the necessity for CEO phase stabilization.
For elliptical polarization, relative to circular polarization, the problem is more complex. The radial momentum $p_{\mathrm{r}}$ follows the envelope of the electric field with oscillations stemming from the ellipticity ${ }^{1}$. However, the ionization time can still be calculated if the ellipticity and the angular orientation of the polarization ellipse are known (Fig. 2).

The electron correlation spectrum for the radial momentum component is expected to show peaks that correspond to the averaged first and second ionization time, see Fig. $2 \mathrm{a}$. In the case of two detected electrons there is no a priori information about which is the first and which is the second released electron, therefore both possibilities are considered and thus the spectrum is symmetric around the first diagonal. For the ionization times presented in Fig. 3 we sorted the electrons by assuming that the first emitted electron carries the smaller radial momentum.

It is important to note that the 3-particle-coincidence detection method does not detect all events with the same efficiency. 
a
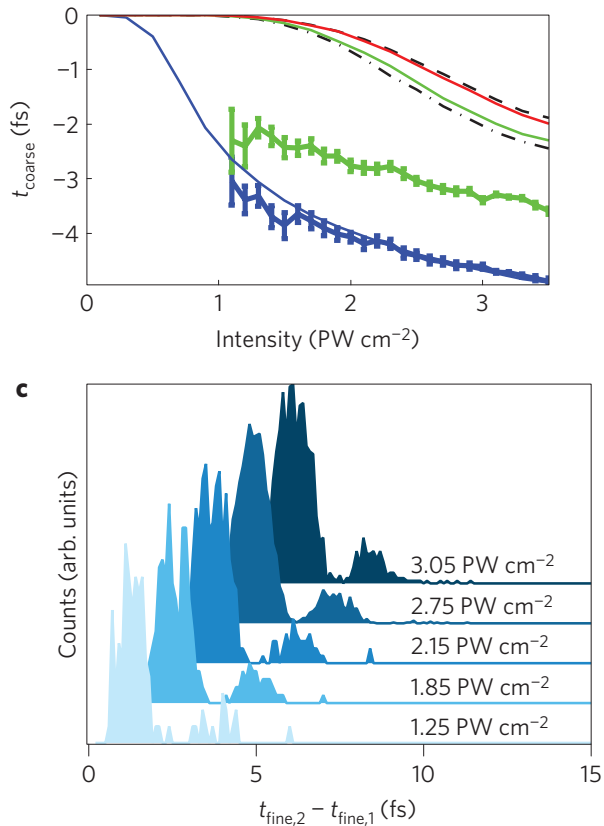

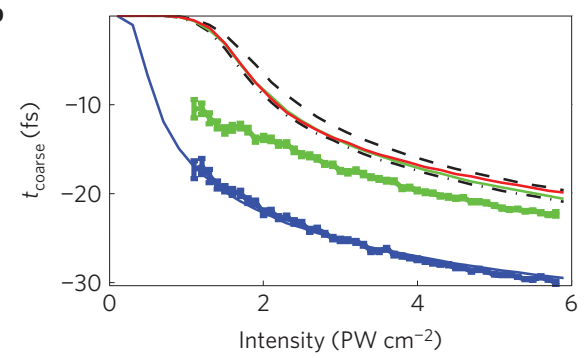

d

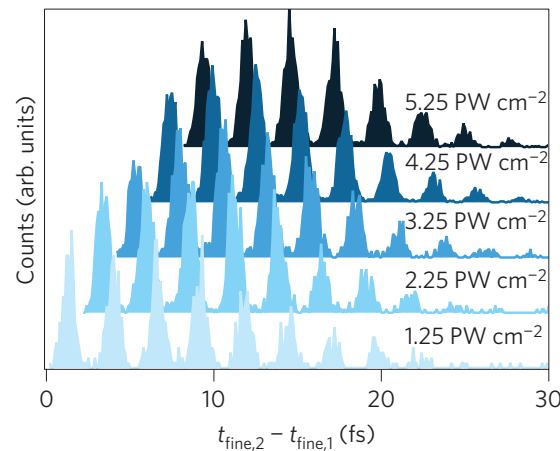

Figure 3 | The ionization times of the first and the second released electron. a, The coarse estimate for the first (blue) and the second (green) ionization time for the 7-fs pulse. The mean value and the statistical error (which is the standard deviation divided by the square root of the sample size) are displayed for different intensities by the solid line with error bars. The SDI simulation (see 'Simulation details') for the first ionization time is shown in the blue solid line. The SDI simulation of the second ionization time is shown for the four different assumptions about the electron $m$ numbers: green solid for (1), black dashed for (2), black dash-dotted for (3), red solid for (4). b. Same as a for the 33-fs pulse. c, For selected intensities from a, the distribution of the time difference between the fine estimates for the ionization times. As electrons flying in the same direction as the $x$ axis are not detected, there are no counts at even multiples of the optical cycle. d, Same as $\mathbf{c}$ for the 33-fs pulse.

The electron-electron correlation spectrum for $p_{\varphi}$ is expected to peak at $\left(0^{\circ}, 0^{\circ}\right)$ and $\left( \pm 180^{\circ}, \pm 180^{\circ}\right)$ for parallel electron emission and at $\left( \pm 180^{\circ}, 0^{\circ}\right)$ and $\left(0^{\circ}, \pm 180^{\circ}\right)$ for anti-parallel electron emission. As can be seen in Fig. $2 b$, practically no electron pairs stemming from parallel emission are detected. The reason for that is the multi-hit dead time of the delay line detector (DLD). Parallel electron emission leads to a short time difference between the electron impacts onto the DLD and therefore low detection efficiency. For anti-parallel electron emission the impact time difference is rather long because the electrons fly in opposite directions after the laser pulse before the extraction field of the spectrometer steers them both towards the electron detector.

\section{Early release of the second electron}

The calculation of the ionization time is done for each laser shot and the results are shown in Fig. 3. For the coarse estimate the mean of the ionization time as a function of intensity is depicted, both for the first and the second ionization step. For the fine estimate the distribution of times between the first and the second ionization step is plotted as a histogram for selected intensities. Strong peaks show up in the histogram at uneven multiples of the optical half-cycle, as expected owing to the ellipticity. The resolution of these peaks is direct evidence for the sub-cycle resolution of the minute hand. The peaks at even multiples of the optical half-cycle are missing, because of the low detection efficiency for parallel electron emission. However it is unlikely that this detection asymmetry affects the value of the averaged ionization times by more than about 20 as, see Supplementary Discussion.

For the first ionization step we find a reasonable agreement of data and SDI simulation in the coarse estimate, both for the 7 -fs and for the 33-fs laser pulse, but for the second ionization step we find a strong deviation (Fig. 3a,b).

An elementary assumption of our SDI model is that the first ionization step produces a ground state ion, which subsequently in the next ionization step is ionized into the ground state of the doubly charged ion. For molecules, where ionization can occur via multiple pathways ${ }^{15}$, this assumption will not hold. For the present case of a noble gas the energy values for excited states with different principal quantum number are energetically so high above the ground state that ionization into them is negligible. States with different $m$ quantum numbers, however, are close in energy and hence will contribute.

The ionization rate, which is responsible for the ionization times in the SDI simulation, depends on the absolute value of the $m$ quantum numbers ${ }^{16}$. Here we considered different options for the distribution of the five valence electrons over the $m$ quantum numbers in $\mathrm{Ar}^{+}$. In the first option (see Simulation details) the electrons are equally distributed among the available $m$ quantum numbers ${ }^{17}$. Any possible correlation between the ionization steps is excluded in this model. The second option (one electron with $|m|=0$ and four electrons with $|m|=1$ ) and third option (two electrons with $|m|=0$ and three electrons with $|m|=1$ ) give the limiting cases for the influence of the $m$ number distribution on the ionization time. Goulielmakis and co-workers found that tunnel ionization launches a coherent process in the valence shell of the remaining ion ${ }^{18}$. The fine structure states of $\mathrm{Ar}^{+}$add coherently and form an electron hole that oscillates with the period given by the spin-orbit splitting. As a consequence, the population of the $m$ numbers also oscillates, and is the fourth option of our SDI simulation. In these options the SDI model fails to describe the timing of the second ionization step (Fig. 3).

For all of the above options of a modified SDI model there is no possibility of electron correlation before the first electron is emitted. There are of course more options for how the SDI model could be further modified after the first ionization step. We speculate that the vicinity of the first ejected electron might trigger the observed early second ionization step. It was shown recently that the departing electron affects the rearrangement of the 
a

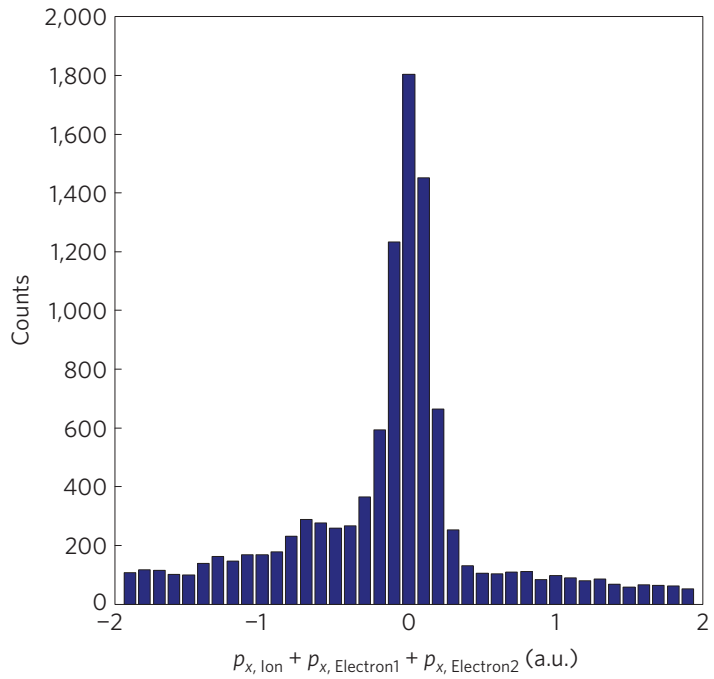

C

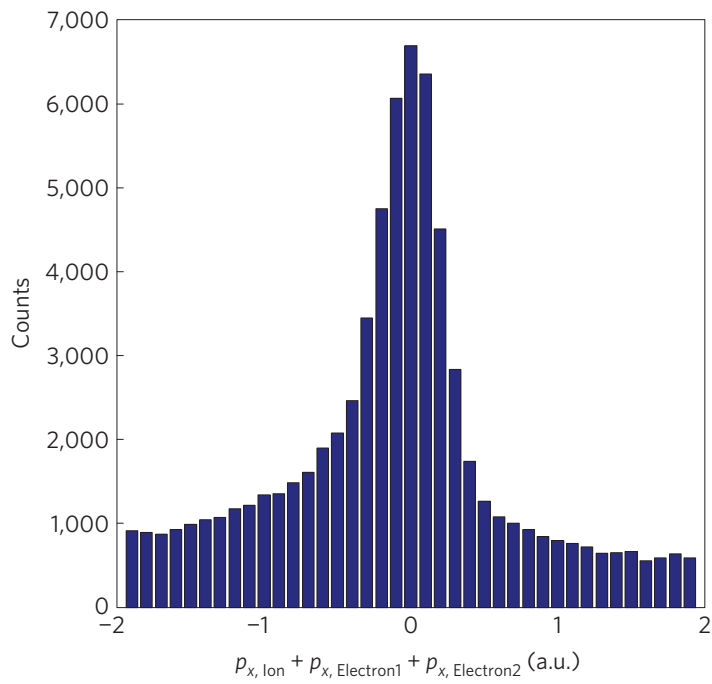

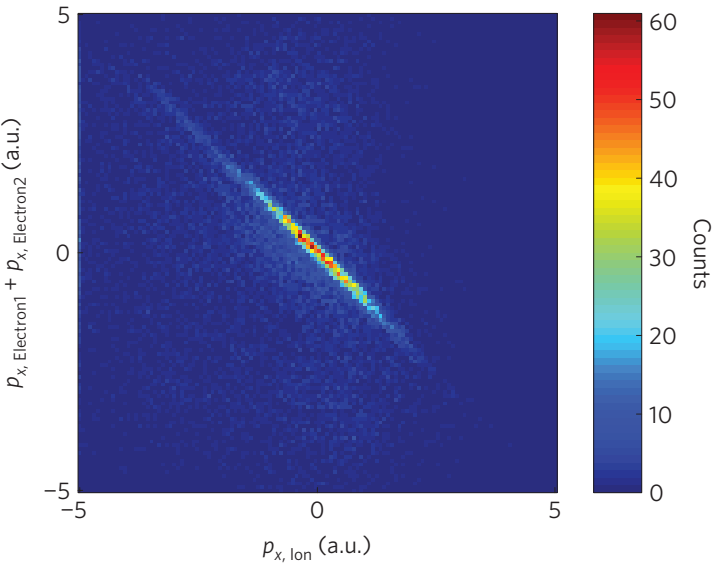

d

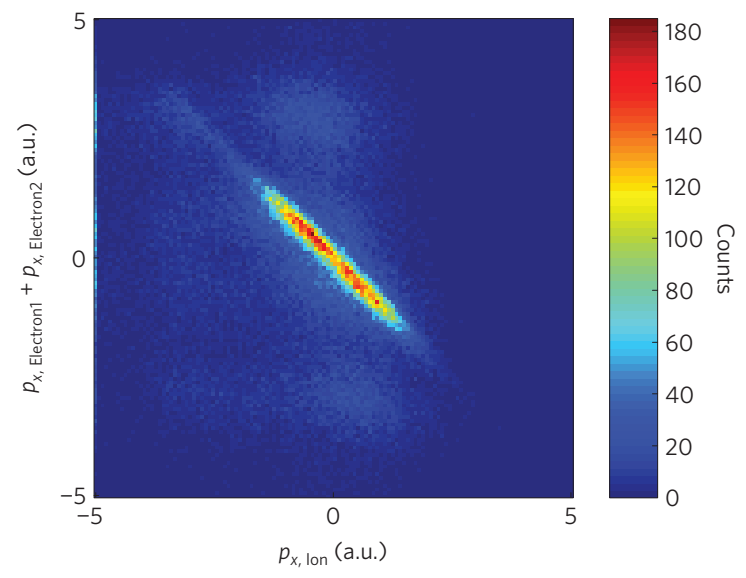

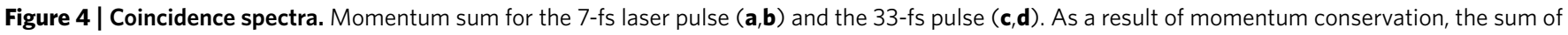
the ion momentum and the two electron momenta must equal zero if the detected particles stem from the breakup of a single atom. Out of all detected

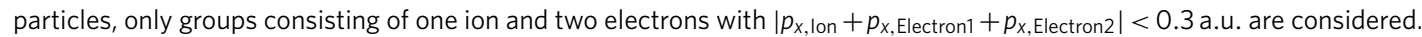

remaining electron cloud in molecules ${ }^{19}$. We believe that also in atoms the first emitted electron may influence the parent ion, such that the averaged ionization probability for the second ionization step is higher than predicted by SDI. An enhanced ion count rate of doubly charged magnesium ions in circularly polarized light has already been reported ${ }^{20}$. However, the count rate statistics of this measurement is insufficient to make an equivalent statement about our measurement.

It is interesting to note that at this point we have neglected the Coulomb interaction between the ion and the electron during the acceleration by the laser field. We have recently discovered that a semi-classical Coulomb correction, as applied before in the tunnelling delay time measurements ${ }^{2}$, fails in the intensity regime of this study (this will be described in a following publication). The actual Coulomb correction is much less significant and cannot explain the observed timing difference described here.

\section{Conclusion}

In conclusion, we have used the electron momentum after ionization by elliptically polarized strong laser pulses as the 'hands' of the attoclock. We used this attoclock to measure the semiclassical ionization times in double ionization of argon. We were able to track how the averaged ionization time moved towards the beginning of the pulse with increasing intensity as a result of depletion. As a consequence of that, the ion momentum distribution projected onto the minor polarization axis shows a bifurcation from a 3-peak into a 4-peak structure. It was shown how the attoclock provides time measurements on two different scales: a coarse timing that supports absolute time measurements without the requirement of CEO phase stabilization, and a fine timing for the time difference of the two ionization steps. In addition, we showed that our SDI tunnelling model, assuming uncorrelated electron emission, seems to be insufficient to describe the second ionization step for elliptically polarized laser pulses. Its predicted second ionization time is too early. It will be some time until this process is fully understood on the basis of quantum mechanical models, but recently there has been significant progress on the basis of fully-classical simulations. These models are able to reproduce many features found experimentally in double ionization by linear light, such as the famous knee-structure ${ }^{21}$, and they are now progressing to describe double ionization by elliptical light $t^{22,23}$.

\section{Methods}

Experimental details. We performed experiments with both a 33-fs laser pulse with a centre wavelength of $788 \mathrm{~nm}$ and a 7 -fs laser pulse with a centre wavelength of $740 \mathrm{~nm}$ produced by a Ti:Sapphire based laser system, and in the latter case 
compressed by a two-stage filament compressor ${ }^{24}$. We continuously varied the intensity of the 33-fs pulse by means of a rotating half-wave plate in front of a polarizer and the intensity of the 7 -fs pulse by means of a motorized iris. For the 33 -fs pulse the angle of the half-wave plate determines the intensity up to a multiplicative factor; the calibration of this factor as well as the intensity calibration of the 7 -fs pulse rely on the $\mathrm{Ar}^{++}$to $\mathrm{Ar}^{+}$count ratio. A COLTRIMS apparatus ${ }^{25}$ measures the momenta of the doubly charged ion and the two electrons arising from double ionization of argon. Polarization control is achieved by using achromatic half-wave and quarter-wave plates. We measured the ellipticity and the angular orientation of the polarization ellipse by measuring the power after a rotating polarizer. The major axis of the polarization ellipse was aligned along the $y$ axis of our reference frame, which is also the direction of the gas jet in the COLTRIMS; the ellipticity was 0.78 for the 7 -fs pulse and 0.77 for the 33 -fs pulse.

We detect one ion and two electrons in 3-particle-coincidence, which means that for each laser shot we select groups consisting of one ion and two electrons out of all detected particles according to the condition $p_{\text {ion }}+p_{\text {electron } 1}+p_{\text {electron } 2} \approx 0$, see Fig. 4 . This ensures that false coincidences, that is particle groups that do not stem from the fragmentation of one atom, are kept low (in our data about $10 \%$ for low intensities and up to $20 \%$ for high intensities).

Simulation details. In our model we assume the electric field is given by

$$
\mathbf{E}(t)=f(t)\left[\frac{\varepsilon}{\sqrt{\varepsilon^{2}+1}} \cos \left(\omega t+\varphi_{\mathrm{CEO}}\right) \widehat{\mathbf{e}}_{\mathbf{x}}+\frac{1}{\sqrt{\varepsilon^{2}+1}} \sin \left(\omega t+\varphi_{\mathrm{CEO}}\right) \widehat{\mathbf{e}}_{\mathrm{y}}\right]
$$

where $f(t)$ is the field envelope, $\varepsilon$ is the ellipticity and $\varphi_{\text {CEO }}$ is the CEO phase $e^{14}$. As static field ionization rates derived from tunnelling theories are known to be invalid in the higher intensity regime, we assume an ionization rate $W$ as proposed by Tong and $\operatorname{Lin}^{11}$. The ionization rate of a single electron depends on the absolute value of its magnetic quantum number $m$. In the ground state of Ar there are two electrons with $m=0$ and four electrons with $|m|=1$ in the outer shell. For the ionization of $\mathrm{Ar}^{+}$(the second ionization step) we consider the following distributions of the five electrons over the $m$ states: (1) equal distribution over the possible $m$ states, (2) one electron with $|m|=0$ and four electrons with $|m|=1$, (3) two electrons with $|m|=0$ and three electrons with $|m|=1$, (4) a beating of (2) and (3) with a period of $23.3 \mathrm{fs}$. Option (4) reflects the coherent superposition of the two fine-structure states $\left(3 s^{2} 3 p^{5}\right)^{2} \mathbf{P}_{1.5}$ and $\left(3 s^{2} 3 p^{5}\right)^{2} \mathbf{P}_{0.5}$, which are separated by $0.177 \mathrm{eV}$ (ref. 18)

When the electric field strength increases, ionization occurs at a rate high enough for saturation to become important. This is accounted for by weighting the ionization rate with the survival probability:

$$
W_{\text {sat }}(t)=W(t) \exp \left(-\int_{t_{0}}^{t} W\left(t^{\prime}\right) \mathrm{d} t^{\prime}\right)
$$

where $t_{0}=-\infty$ for the first ionization step and $t_{0}=t_{1}$ for the second ionization step if the first electron was ionized at $t_{1}$. $W_{\text {sat }}$ is essentially confined to a time interval in the beginning of the pulse (Fig. $2 a$ ).

After ionization the electron is assumed to be released into the continuum with zero kinetic energy and subsequently accelerated by the laser field, as in the three-step model ${ }^{10}$, such that the final momentum after the laser pulse of an electron that was freed at a time $t$ can be expressed as

$$
\mathbf{p}(t)=-\mathbf{A}(t)
$$

(atomic units are used throughout the paper) where $\mathbf{A}$ is the vector potential defined as

$$
\begin{aligned}
\mathbf{A}(t) & =\int_{t}^{\infty} \mathbf{E}\left(t^{\prime}\right) \mathrm{d} t^{\prime} \\
& \approx \frac{f(t)}{\omega}\left[-\frac{\varepsilon}{\sqrt{\varepsilon^{2}+1}} \sin \left(\omega t+\varphi_{\text {CEO }}\right) \widehat{\mathbf{e}}_{\mathbf{x}}+\frac{1}{\sqrt{\varepsilon^{2}+1}} \cos \left(\omega t+\varphi_{\text {CEO }}\right) \widehat{\mathbf{e}}_{\mathrm{y}}\right]
\end{aligned}
$$

We implemented the model as a Monte Carlo simulation, averaging over all possible CEO phases because in the experiment the CEO phase was not stabilized. For a given peak intensity, averaging over the intensity distribution of the focal volume is done assuming a Gaussian beam profile.

Received 19 October 2010; accepted 8 January 2011; published online 20 March 2011

\section{References}

1. Eckle, P. et al. Attosecond angular streaking. Nature Phys. 4, 565-570 (2008).

2. Eckle, P. et al. Attosecond ionization and tunnelling delay time measurements in helium. Science 322, 1525-1529 (2008).

3. Weber, T. et al. Correlated electron emission in multiphoton double ionization. Nature 405, 658-661 (2000).

4. Rudenko, A. et al. Correlated multielectron dynamics in ultrafast laser pulse interactions with atoms. Phys. Rev. Lett. 93, 253001 (2004).

5. Becker, W. \& Rottke, H. Many-electron strong-field physics. Contemp. Phys. 49, 199-223 (2008).

6. Dörner, R. et al. in Advances in Atomic, Molecular, and Optical Physics, Vol. 48 (eds Bederson, B. \& Walther, H.) 1-34 (Academic, 2002).

7. Maharian, C. M. et al. Momentum imaging of doubly charged ions of $\mathrm{Ne}$ and Ar in the sequential ionization region. Phys. Rev. A 72, 041403 (2005).

8. Smolarski, M., Eckle, P., Keller, U. \& Dörner, R. Semiclassical model for attosecond angular streaking. Opt. Express 18, 17640-17650 (2010).

9. Wang, X. \& Eberly, J. H. Effects of elliptical polarization on strong-field short-pulse double ionization. Phys. Rev. Lett. 103, 103007 (2009).

10. Corkum, P. B. Plasma perspective on strong-field multiphoton ionization. Phys. Rev. Lett. 71, 1994-1997 (1993).

11. Tong, X. M. \& Lin, C. D. Empirical formula for static field ionization rates of atoms and molecules by lasers in the barrier-suppression regime. J. Phys. B 38, 2593-2600 (2005).

12. Haessler, S. et al. Phase-resolved attosecond near-threshold photoionization of molecular nitrogen. Phys. Rev. A 80, 011404 (2009).

13. Schultze, M. et al. Delay in photoemission. Science 328, 1658-1662 (2010).

14. Telle, H. R. et al. Carrier-envelope offset phase control: A novel concept for absolute optical frequency measurement and ultrashort pulse generation. Appl. Phys. B 69, 327-332 (1999).

15. Litvinyuk, I. V. et al. Shakeup excitation during optical tunnel ionization. Phys. Rev. Lett. 94, 033003 (2005).

16. Ammosov, M. V., Delone, N. B. \& Krainov, V. P. Tunnel ionization of complex atoms and of atomic ions in an alternating electromagnetic field. Sov. Phys. JETP 64, 1191-1194 (1986).

17. Taieb, R., Veniard, V. \& Maquet, A. Photoelectron spectra from multiple ionization of atoms in ultra-intense laser pulses. Phys. Rev. Lett. 87, 053002 (2001).

18. Goulielmakis, E. et al. Real-time observation of valence electron motion. Nature 466, 739-743 (2010).

19. Walters, Z. B. \& Smirnova, O. Attosecond correlation dynamics during electron tunnelling from molecules. J. Phys. B 43, 161002 (2010).

20. Gillen, G. D., Walker, M. A. \& Van Woerkom, L. D. Enhanced double ionization with circularly polarized light. Phys. Rev. A 64, 043413 (2001).

21. Fittinghoff, D. N., Bolton, P. R., Chang, B. \& Kulander, K. C. Observation of nonsequential double ionization of helium with optical tunneling. Phys. Rev. Lett. 69, 2642-2645 (1992).

22. Mauger, F., Chandre, C. \& Uzer, T. Recollisions and correlated double ionization with circularly polarized light. Phys. Rev. Lett. 105, 083002 (2010).

23. Wang, X. \& Eberly, J. H. Elliptical polarization and probability of double ionization. Phys. Rev. Lett. 105, 083001 (2010).

24. Hauri, C. P. et al. Generation of intense, carrier-envelope phase-locked few-cycle laser pulses through filamentation. Appl. Phys. B 79, 673-677 (2004).

25. Dörner, R. et al. Cold target recoil ion momentum spectroscopy: A 'momentum microscope' to view atomic collision dynamics. Phys. Rep. 330, 95-192 (2000).

\section{Acknowledgements}

This work was supported by the NCCR Quantum Photonics (NCCR QP), a research instrument of the Swiss National Science Foundation (SNSF), by ETH Research Grant ETH-03 09-2 and by the SNSF R'Equip grant 206021_128551/1. R.D. acknowledges support by a Koselleck Project of the Deutsche Forschungsgemeinschaft. We thank H. J. Wörner for enlightening discussions.

\section{Author contributions}

A.N.P. and C.C. performed the experiments. A.N.P. analysed the data and implemented the simulation. All authors were involved in the interpretation. A.N.P., R.D. and U.K. wrote the manuscript.

\section{Additional information}

The authors declare no competing financial interests. Supplementary information accompanies this paper on www.nature.com/naturephysics. Reprints and permissions information is available online at http://npg.nature.com/reprintsandpermissions. Correspondence and requests for materials should be addressed to A.N.P. 